\title{
Microstructure of a near-critical colloidal dispersion under stationary shear flow
}

\author{
Hao Wang, M Pavlik Lettinga and Jan K G Dhont \\ Research Centre Jülich, IFF, Institut Weiche Materie, D-52425 Jülich, Germany
}

Received 25 June 2002

Published 9 August 2002

Online at stacks.iop.org/JPhysCM/14/7599

\begin{abstract}
Microstructural order of near-critical suspensions of colloid-polymer mixtures under stationary shear flow is investigated by means of small-angle light scattering. The experiments reveal a significant, unexpected shear-induced distortion in directions perpendicular to the flow direction, the more so on closer approach to the gas-liquid critical point. Starting from the $N$-particle Smoluchowski equation, we derive an explicit expression for the shear-rate dependence of the static structure factor. The present theory improves on a previous treatment, by taking the shear-induced distortions of short-ranged correlations into account, which are indeed shown to be important on close approach to the critical point. The experiments are in quantitative accordance with this extended theory, for all wavevectors, shear rates, and distances from the gas-liquid critical point.
\end{abstract}

\section{Introduction}

Peter Pusey has been a pioneer in the area of light scattering by colloids, always combining experimental with thorough theoretical insight. It therefore seems appropriate to contribute to the Festschrift on the occasion of his 60th birthday with an experimental light scattering study with a considerable theoretical component. We shall report here on light scattering experiments on colloid-polymer mixtures in the vicinity of the gas-liquid critical point under stationary shear flow.

Close to a gas-liquid critical point, long-ranged correlations exist and the dynamics of collective modes becomes extremely slow. These features render microstructural order close to the critical point very sensitive to shear flow. Relatively small shear rates will have a large effect on the critical, small-wavevector dependence of the structure factor.

Inhomogeneities that extend solely in directions perpendicular to the flow direction remain intact under shear flow. One might therefore expect that in these directions, as compared to other directions, microstructural order will be less affected by shear flow. Indeed, an existing mean-field theory for microstructural order of sheared, near-critical suspensions predicts no change of the structure factor in the plane perpendicular to the shear flow [1,2]. As argued in 
the references cited above (and later in the present paper), finite distortions are expected beyond the mean-field region only on very close approach to the gas-liquid critical point. The nonnegligible distortions of near-critical, long-ranged microstructural order in these directions, as observed by means of small-angle light scattering experiments in [3], are therefore attributed to non-mean-field-like behaviour. It will be shown here, however, that the actual reason for the observed long-ranged distortion of the microstructure in directions perpendicular to the shear flow results from distortion of the microstructural order that extends over distances at most equal to the range of the pair-interaction potential. The study in [3] is of a qualitative nature, which did not allow for a quantitative comparison of light scattering data with theory. The much more accurate small-angle light scattering experiments described in the present paper became feasible after the development of a new optical shear cell, and do allow such a quantitative comparison of the full wavevector dependence of scattering patterns with theory, as a function of shear rate and the distance from the critical point. Short-ranged distortions are shown to couple to long-ranged distortions in a way that leads to a finite distortion of long-ranged, near-critical microstructure in directions perpendicular to the flow direction. Non-mean-field phenomena come into play only when the critical point is approached much more closely, as in the experiments described in [3] and in the present paper. For the colloid-polymer mixtures used in [3] and in the present paper, it is probably not feasible to approach the critical point in a controlled way so closely that these non-mean-field effects become significant.

In order to explain our experimental data on the shear-induced distortion of long-ranged correlations, we need to assume a rather unexpected form for the shear-rate dependence of the short-ranged part of the pair-correlation function, the origin of which is not yet clear. The evidence presented here for this peculiar form is indirect, in the sense that these short-ranged shear-induced structures are probed via their coupling to long-ranged correlations. We are planning to perform neutron scattering experiments that directly probe the short-ranged part of the correlation function for the same system, in order to verify the assumed form for the shear-rate dependence of the pair-correlation function for short distances.

It has been shown in [4] that the distortion of short-ranged correlations is responsible for the shear-induced displacement of the gas-liquid critical point. Hence, the finite distortion of critical microstructure in directions perpendicular to the flow direction can be interpreted as being the result of a shift of the critical point when shear flow is applied. Indeed, it will be shown that for wavevectors perpendicular to the flow direction, the structure factor attains the equilibrium Ornstein-Zernike form, except that the correlation length is now an 'effective correlation length', which is shear-rate dependent and smaller than the correlation length of the quiescent, unsheared suspension.

This paper is organized as follows. In the theoretical section we first summarize the Smoluchowski equation approach used to obtain the shear-distorted pair-correlation function for nearcritical suspensions. It will be shown how distortion of short-ranged correlations, which was neglected before [1,2], affects the long-ranged part of the total-correlation function. The experimental section contains a description of the colloidal system and the set-up used for the measurement of the small-angle, critical part of the structure factor. Experimental data are presented and analysed in terms of the new theory. The paper ends with a summary and conclusions.

\section{Theory}

Quite a few theoretical approaches describing the effects of shear flow on the microstructure of spherical colloids have been proposed [5-10]. We will refer to these theories in appropriate places later in this section. The approach taken in the present section is specific to colloidal suspensions near their gas-liquid critical point. 
The starting point here is the $N$-particle Smoluchowski equation, which is an equation of motion for the probability density function $P$ of the position coordinates $\boldsymbol{r}_{j}, j=1, \ldots, N$, of all $N$ spherical Brownian particles in the system under consideration. The $N$-particle Smoluchowski equation reads

$$
\frac{\partial}{\partial t} P\left(\boldsymbol{r}_{1}, \ldots, \boldsymbol{r}_{N}, t\right)=D_{0} \sum_{j=1}^{N} \nabla_{j} \cdot\left[\nabla_{j} P+\beta P \nabla_{j} \Phi\right],
$$

where $D_{0}$ is the translational diffusion coefficient of a free, non-interacting colloidal sphere, $\nabla_{j}$ is the gradient operator with respect to the position coordinate $\boldsymbol{r}_{j}$ of particle $j, \Phi$ is the total interaction energy of the assembly of $N$ colloidal spheres, and $\beta=1 / k_{B} T$ ( $k_{B}$ is Boltzmann's constant and $T$ is the temperature). In equation (1) we neglected hydrodynamic interactions: the role of hydrodynamic interactions will be discussed later in this section. For the homogeneous fluid of spheres under consideration, the pair-correlation function $g$ is defined as

$$
g\left(\boldsymbol{r}_{1}, \boldsymbol{r}_{2}, t\right)=\frac{1}{V^{2}} \int \mathrm{d} \boldsymbol{r}_{3} \ldots \int \mathrm{d} \boldsymbol{r}_{N} P\left(\boldsymbol{r}_{1}, \boldsymbol{r}_{2}, \boldsymbol{r}_{3}, \ldots, \boldsymbol{r}_{N}, t\right) .
$$

The equation of motion for $g$ can thus be obtained by integration of the $N$-particle Smoluchowski equation. For a pairwise-additive total interaction energy $\Phi$,

$$
\Phi\left(\boldsymbol{r}_{1}, \ldots, \boldsymbol{r}_{N}\right)=\sum_{i>j} V\left(\left|\boldsymbol{r}_{i}-\boldsymbol{r}_{j}\right|\right),
$$

where $V$ is the pair-interaction potential, the resulting stationary equation of motion for the pair-correlation function that is thus found reads $[1,2]$

$$
0=2 D_{0} \boldsymbol{\nabla} \cdot\left[\nabla g(\boldsymbol{r})+\beta g(\boldsymbol{r})\left\{\boldsymbol{\nabla} V(\boldsymbol{r})-\boldsymbol{F}^{\text {ind }}(\boldsymbol{r})\right\}\right]-\boldsymbol{\nabla} \cdot[\boldsymbol{\Gamma} \cdot \boldsymbol{r} g(\boldsymbol{r})],
$$

where $\bar{\rho}=N / V$ is the colloidal particle number density ( $N$ is the number of colloidal particles in the system with volume $V$ ). Furthermore,

$$
\boldsymbol{F}^{\text {ind }}(\boldsymbol{r})=-\bar{\rho} \int \mathrm{d} \boldsymbol{r}^{\prime}\left[\nabla_{r^{\prime}} V\left(r^{\prime}\right)\right] \frac{g_{3}\left(\boldsymbol{r}, \boldsymbol{r}^{\prime}\right)}{g(\boldsymbol{r})},
$$

is the force between the two particles separated by the distance $r$ that is mediated through third particles (the superscript ' $i n d$ ' stands for 'indirect'). Here, $g_{3} \equiv g_{3}\left(\boldsymbol{r}_{1}, \boldsymbol{r}_{2}, \boldsymbol{r}_{3}\right)$ is the three-particle correlation function, where $\boldsymbol{r}_{j}$ is the position coordinate of the $j$ th colloidal particle, with $r=r_{1}-r_{2}$ and $r^{\prime}=r_{1}-r_{3}$. The tensor $\Gamma$ is the velocity gradient tensor, which we choose as

$$
\boldsymbol{\Gamma}=\dot{\gamma} \hat{\boldsymbol{\Gamma}}, \quad \text { with } \hat{\boldsymbol{\Gamma}}=\left(\begin{array}{ccc}
0 & 1 & 0 \\
0 & 0 & 0 \\
0 & 0 & 0
\end{array}\right)
$$

with $\dot{\gamma}$ the shear rate. This externally imposed fluid flow is a flow in the $x$-direction, with its gradient in the $y$-direction.

We are interested here in the asymptotic solution of equation (4) for large distances, that is, for $r \gg R_{V}$, where $R_{V}$ is the range of the pair-interaction potential.

Let us first summarize the steps, described in more detail in [1, 2], that are taken in order to obtain a closed equation of motion from equation (4) for this long-ranged behaviour of the total-correlation function $h=g-1$ : (i) The three-particle correlation function is expressed in terms of pair-correlation functions by means of a modified superposition approximation that was first proposed by Fixman [11]. On using the classic Kirkwood superposition approximation, where $g_{3}\left(\boldsymbol{r}_{1}, \boldsymbol{r}_{2}, \boldsymbol{r}_{3}\right)=g\left(\boldsymbol{r}_{1}-\boldsymbol{r}_{2}\right) g\left(\boldsymbol{r}_{3}-\boldsymbol{r}_{2}\right) g\left(\boldsymbol{r}_{1}-\boldsymbol{r}_{3}\right)$, or equivalently, $g_{3}\left(\boldsymbol{r}, \boldsymbol{r}^{\prime}\right)=g(\boldsymbol{r}) g\left(\boldsymbol{r}-\boldsymbol{r}^{\prime}\right) g\left(\boldsymbol{r}^{\prime}\right)$, it turns out (on going through the entire analysis as described below) that the correlation length remains finite, right up to the critical point. The Kirkwood 


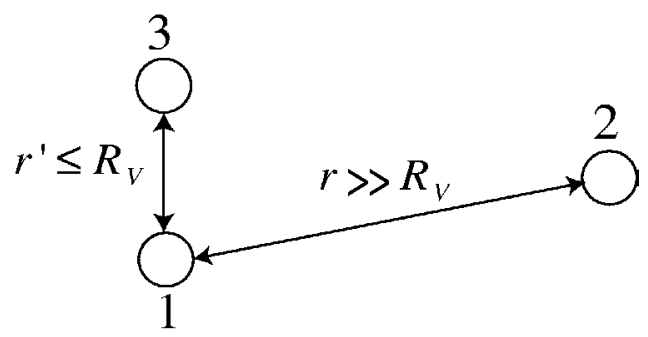

Figure 1. A sketch of the configurations that are of interest for the closure relation for $g_{3}$ in the indirect force $\boldsymbol{F}^{\text {ind }}(\boldsymbol{r})$ in equation (5), where $\boldsymbol{r}=\boldsymbol{r}_{1}-\boldsymbol{r}_{2}$ and $\boldsymbol{r}^{\prime}=\boldsymbol{r}_{1}-\boldsymbol{r}_{3}$. The particles 1 and 2 are far apart-that is, $r \gg R_{V}$ - while particles 1 and 3 are within a distance $f^{\prime} \leqslant R_{V}$.

superposition approximation is therefore a very bad approximation in the vicinity of the gasliquid spinodal. The point here is that $r=\left|\boldsymbol{r}_{1}-\boldsymbol{r}_{2}\right| \gg R_{V}$, while $r^{\prime}=\left|\boldsymbol{r}_{1}-\boldsymbol{r}_{3}\right| \leqslant R_{V}$ in the integral in equation (5), due to the presence of the multiplicative contribution $\mathrm{d} V\left(r^{\prime}\right) / \mathrm{d} r^{\prime}$ in the integrand. That is, the three-particle configurations that matter for the closure relation of $g_{3}$ are those where particles 1 and 2 are separated by a distance much larger than $R_{V}$, while particles 1 and 3 are close together, to within the range $R_{V}$ of the pair potential, as sketched in figure 1 . The effect of the presence of the well separated particle on the pair-correlation function $g\left(\boldsymbol{r}^{\prime}\right)$ between the two neighbouring particles is that it enhances the local density in the vicinity of these two particles by an amount $\bar{\rho} h\left(\boldsymbol{r}-\frac{1}{2} \boldsymbol{r}^{\prime} \mid \dot{\gamma}\right)$. An improved superposition approximation, which should be referred to as 'the Kirkwood-Fixman closure relation', thus reads

$$
g_{3}\left(\boldsymbol{r}, \boldsymbol{r}^{\prime}\right)=g(\boldsymbol{r}) g\left(\boldsymbol{r}-\boldsymbol{r}^{\prime}\right) g^{*}\left(\boldsymbol{r}^{\prime}\right),
$$

where the $*$ indicates that the correlation function is to be evaluated at the density

$$
\rho^{*}=\bar{\rho}+\bar{\rho} h\left(\boldsymbol{r}-\frac{1}{2} \boldsymbol{r}^{\prime}\right) .
$$

This modified superposition approximation does indeed lead to the correct divergence of the correlation length on approach of the critical point.

(ii) Since the total-correlation function $h=g-1 \rightarrow 0$ as $r \rightarrow \infty$, its asymptotic behaviour at distances $r \gg R_{V}$ is obtained by linearization of the equation of motion equation (4) with respect to the long-ranged part of total-correlation function. For example, the closure relation equations (7), (8) can be written as

$$
g^{*}\left(\boldsymbol{r}^{\prime}\right)=g\left(\boldsymbol{r}^{\prime}\right)+\frac{\mathrm{d} g\left(\boldsymbol{r}^{\prime}\right)}{\mathrm{d} \bar{\rho}} \bar{\rho} h\left(\boldsymbol{r}-\frac{1}{2} \boldsymbol{r}^{\prime}\right),
$$

which is just a leading-order Taylor expansion.

(iii) Assuming that for $r \gg R_{V}$, the correlation functions are smooth on the length scale set by $R_{V}$, we can Taylor expand $g\left(r-r^{\prime}\right)$ and $h\left(r-\frac{1}{2} r^{\prime}\right)$ (which appear in the integral in equation (5) after performing steps (i) and (ii)) in a gradient series with respect to $\boldsymbol{r}^{\prime}$, up to third-order gradients.

(iv) As a last step, the pair-correlation function $g\left(\boldsymbol{r}^{\prime}\right)$ in equation (9) is set equal to the equilibrium pair-correlation function $g^{e q}\left(r^{\prime}\right)$ - that is, the correlation function in the absence of shear flow. The reason for neglecting the shear-rate dependence of this correlation function is as follows. In the integral in equation (5), only those $r^{\prime}$ with $r^{\prime} \leqslant R_{V}$ contribute. Shearinduced distortion of the pair-correlation function for these short distances $r^{\prime}$ is measured by the bare Peclet number:

$$
P e^{0}=\frac{\dot{\gamma} R_{V}^{2}}{2 D_{0}}
$$


As will be seen later, the amount of distortion of the long-ranged correlations is measured by an effective Peclet number:

$$
\lambda=\frac{\dot{\gamma} \xi^{2}}{2 D^{e f f}},
$$

where $\xi$ is the correlation length of the quiescent, unsheared suspension, and $D^{\text {eff }}$ is an effective diffusion coefficient (which will be specified later). Near the critical point, $\xi \gg R_{V}$ and $D^{e f f} \ll D_{0}$, due to critical slowing down. Hence, even though $\lambda$ can be very large, the bare Peclet number is usually fairly small. This quantifies the intuition that slowly fluctuating, large-scale structures are more easily affected by shear flow than rapidly fluctuating, small-scale structures. Since we are interested here in the distortion of long-ranged, critical microstructure, it seems appropriate to neglect the distortion of short-ranged correlations - that is, to set $g\left(\boldsymbol{r}^{\prime}\right)$ in equation (9) equal to $g^{e q}\left(r^{\prime}\right)$.

Performing angular integrations, these steps transform equation (4) for $r \gg R_{V}$ into

$$
0=2 \beta D_{0} \nabla \cdot\left[\frac{\mathrm{d} \Pi}{\mathrm{d} \bar{\rho}} \nabla h(\boldsymbol{r})-\Sigma \nabla \nabla^{2} h(\boldsymbol{r})\right]-\nabla \cdot[\boldsymbol{\Gamma} \cdot \boldsymbol{r} h(\boldsymbol{r})]
$$

up to ' $\mathcal{O}\left(\left(R_{V} \nabla\right)^{6}\right)$ '. Here $\Pi$ is the osmotic pressure:

$$
\Pi=\beta^{-1} \bar{\rho}-\frac{2 \pi}{3} \bar{\rho}^{2} \int_{0}^{\infty} \mathrm{d} r^{\prime} r^{\prime 3} \frac{\mathrm{d} V\left(r^{\prime}\right)}{\mathrm{d} r^{\prime}} g^{e q}\left(r^{\prime}\right),
$$

and $\Sigma$ is a positive constant, proportional to the Cahn-Hilliard square gradient coefficient:

$$
\Sigma=\frac{2 \pi}{15} \bar{\rho} \int_{0}^{\infty} \mathrm{d} r^{\prime} r^{\prime 5} \frac{\mathrm{d} V\left(r^{\prime}\right)}{\mathrm{d} r^{\prime}}\left(g^{e q}\left(r^{\prime}\right)+\frac{1}{8} \bar{\rho} \frac{\mathrm{d} g^{e q}\left(r^{\prime}\right)}{\mathrm{d} \bar{\rho}}\right) .
$$

Fourier transformation of equation (12) leads to the following equation of motion for the structure factor $S(\boldsymbol{k})$, where $\boldsymbol{k}$ is the Fourier variable conjugate of $\boldsymbol{r}$, which is the scattering wavevector in light scattering experiments:

$$
\dot{\gamma} k_{1} \frac{\partial S(\boldsymbol{k})}{\partial k_{2}}=2 D^{e f f}(k) k^{2}\left\{S(\boldsymbol{k})-S^{e q}(k)\right\},
$$

where $k_{j}$ is the $j$ th component of the wavevector (with ' $j=1$ ' the flow direction, ' $j=2$ ' the gradient direction, and ' $j=3$ ' the vorticity direction), and

$$
D^{e f f}(k)=D_{0} \beta\left[\frac{\mathrm{d} \Pi}{\mathrm{d} \bar{\rho}}+k^{2} \Sigma\right],
$$

is an effective diffusion coefficient. Furthermore,

$$
S^{e q}(k)=\frac{1}{\beta \Sigma} \frac{1}{\xi^{-2}+k^{2}},
$$

is the equilibrium Ornstein-Zernike structure factor, with

$$
\xi=\sqrt{\Sigma / \frac{\mathrm{d} \Pi}{\mathrm{d} \bar{\rho}}}
$$

the correlation length of the quiescent, unsheared system. Note that since $\mathrm{d} \Pi / \mathrm{d} \bar{\rho} \rightarrow 0$ on approach to the critical point, the correlation length diverges. This would not have been found by simply using the original Kirkwood superposition approximation.

Equation (15) can be written in dimensionless form, by introducing the dimensionless wavevector

$$
\boldsymbol{K}=\boldsymbol{k} \xi
$$


as

$$
\lambda K_{1} \frac{\partial S(\boldsymbol{K})}{\partial K_{2}}=K^{2}\left(1+K^{2}\right)\left\{S(\boldsymbol{K})-S^{e q}(K)\right\},
$$

where $\lambda$ is the dressed Peclet number that was already introduced in equation (11) (with $D^{e f f}=D^{e f f}(k=0)$ ), which can also be written, according to equations (16), (18), as

$$
\lambda=\frac{\dot{\gamma} \xi^{2}}{2 D^{e f f}}=\frac{\dot{\gamma} \xi^{4}}{2 D_{0} \beta \Sigma} .
$$

The equation of motion (20) can be solved analytically [1,2]. The analytic solution is very similar, but not entirely identical, to an expression derived by Ronis [6] on the basis of a fluctuating diffusion equation.

As can be seen from equation (20),

$$
S(\boldsymbol{K})=S^{e q}(K) \quad\left(K_{1}=0\right) .
$$

That is, in directions perpendicular to the flow direction no effect of shear flow is predicted. In these directions the structure factor is predicted to retain its equilibrium Ornstein-Zernike form. As will be shown in the experimental section, the structure factor is not found to retain its equilibrium form in directions perpendicular to the flow direction, the more so on closer approach to the critical point, contrary to the prediction (22). Such finite distortion has also been found in [3], where, due to relatively large experimental errors, a significant distortion could be detected only on very close approach to the critical point. There, the finite distortion was attributed to failure of the linearization of the equation of motion for $h$ (step (ii) discussed above). The argument for such a conjecture is as follows. In expanding with respect to $h$, it turns out that one neglects terms of the form $\sim h^{2}$ against a leading term of the form $\beta h \mathrm{~d} \Pi / \mathrm{d} \bar{\rho}$. Hence, one actually neglects $h(r)$ for $r \gg R_{V}$ against $\beta \mathrm{d} \Pi / \mathrm{d} \bar{\rho}$. Since the latter quantity tends to 0 on approach to the critical point, such a leading-order expansion does not suffice any longer very close to the critical point. The region around the critical point where $\beta \mathrm{d} \Pi / \mathrm{d} \bar{\rho}$ is still large enough for one to perform a leading-order expansion with respect to $h$ is referred to as the 'mean-field region'. Contributions non-linear in $h$ to the equation of motion for $h$ should be taken into account on very close approach of the critical point, beyond the mean-field region. Here, non-linear equations of motion must be considered. This led to the conjecture in [3] that the observed distortion on close approach of the critical point was due to non-mean-field behaviour. In the present study, where much more accurate light scattering experiments are presented, we found a significant distortion already occurring relatively far away from the critical point (which could not be seen in the previous light scattering study due to the much larger experimental errors).

The neglect of hydrodynamic interactions between the colloidal particles might be one reason for the discrepancy between the experiments and the prediction of equation (22). Hydrodynamic interactions can be included on the Oseen level—which would be the most important contribution for the large-scale structures that we are interested in here. However, we found that such hydrodynamic interaction contributions are quite small, and are certainly not responsible for the discrepancy.

The explanation for the discrepancy is hidden in the assumption (iv), described above, where the short-ranged part of the pair-correlation function $g\left(r^{\prime}\right)$ is taken equal to its equilibrium form. As will become clear below, the contribution $\sim P e^{0}$ arising from shortranged distortions should be compared to contributions $\sim \beta \mathrm{d} \Pi / \mathrm{d} \bar{\rho}$ (just like the contributions non-linear in $h$ as discussed above). Hence, even though the bare Peclet number $P e^{0}$ is small, it can generally not be neglected, since $\beta \mathrm{d} \Pi / \mathrm{d} \bar{\rho} \rightarrow 0$ on approach of the critical point. We shall therefore expand $g\left(\boldsymbol{r}^{\prime}\right)$ up to leading order in the bare Peclet number (10); that is,

$$
g\left(r^{\prime}\right)=g^{e q}\left(r^{\prime}\right)\left[1+P e^{0} g_{1}\left(r^{\prime}\right)+\mathcal{O}\left(\left(P e^{0}\right)^{2}\right)\right] \quad\left(r^{\prime} \leqslant R_{V}\right) .
$$


Since the function $g_{1}$ will be only slightly non-spherically symmetric, it suffices to expand up to second-order spherical harmonics; that is,

$$
g_{1}\left(\boldsymbol{r}^{\prime}\right)=f_{0}\left(r^{\prime}\right)+\hat{\boldsymbol{r}}^{\prime} \cdot \mathbf{H} \cdot \hat{\boldsymbol{r}}^{\prime} f_{1}\left(r^{\prime}\right) \quad\left(r^{\prime} \leqslant R_{V}\right),
$$

where $\hat{\boldsymbol{r}}^{\prime}=\boldsymbol{r}^{\prime} / \boldsymbol{r}^{\prime}$. The first-order harmonic contribution is absent, since $q\left(\boldsymbol{r}^{\prime}\right)$ must be invariant under the coordinate inversion $\boldsymbol{r}^{\prime} \rightarrow-\boldsymbol{r}^{\prime}$. The tensor $\mathbf{H}$ may be taken as symmetric (since its anti-symmetric part does not contribute to the form $\hat{\boldsymbol{r}}^{\prime} \cdot \mathbf{H} \cdot \hat{\boldsymbol{r}}^{\prime}$ ), while the components $H_{j 3}$ with $j \neq 3$ are 0 due to invariance of $q$ under the transformation $z^{\prime} \rightarrow-z^{\prime}$. Furthermore, without loss of generality, $\mathbf{H}$ may be taken traceless, since a non-zero trace may be absorbed into the function $f_{0}$. Hence, $\mathbf{H}$ is of the form

$$
\mathbf{H}=\left(\begin{array}{ccc}
m_{1} & 1 & 0 \\
1 & m_{2} & 0 \\
0 & 0 & -m_{1}-m_{2}
\end{array}\right) .
$$

As will be found in the next section, the diagonal elements of $\mathbf{H}$ in equation (25) are not needed to explain the experiments. We shall therefore infer, without proof, that $m_{1}=0=m_{2}$. However, the isotropic contribution $f_{0}$ in equation (24) will be essential. We are thus left with

$$
g\left(\boldsymbol{r}^{\prime}\right)=g^{e q}\left(r^{\prime}\right)\left[1+P e^{0}\left\{f_{0}\left(r^{\prime}\right)+\hat{\boldsymbol{r}}^{\prime} \cdot \hat{\mathbf{E}} \cdot \hat{\boldsymbol{r}}^{\prime} f_{1}\left(r^{\prime}\right)\right\}\right] \quad\left(r^{\prime} \leqslant R_{V}\right),
$$

up to first order in $P e^{0}$, where

$$
\hat{\mathbf{E}}=\frac{1}{2}\left[\hat{\boldsymbol{\Gamma}}+\hat{\boldsymbol{\Gamma}}^{\mathrm{T}}\right]
$$

is the symmetric part of the velocity gradient tensor in equation (6).

One might argue that the isotropic contribution $\sim f_{0}$ should be 0 , since an isotropic contribution should be invariant under reversal of the flow. However, in the experimental section, an isotropic contribution is shown to be essential to explain our experiments. When $f_{0} \equiv 0$, then the next higher-order contribution to the isotropic distortion $\sim\left(P e^{0}\right)^{2}$ should be taken into account in order to explain our experimental findings. Experimental evidence will be given in the next section that the isotropic contribution is linear in $P e^{0}$. In view of the invariance of the isotropic distortion under reversal of the shear rate, instead of equation (26) it seems that the correct expansion reads

$$
g\left(\boldsymbol{r}^{\prime}\right)=g^{e q}\left(r^{\prime}\right)\left[1+\left|P e^{0}\right| f_{0}\left(r^{\prime}\right)+P e^{0} \hat{\boldsymbol{r}}^{\prime} \cdot \hat{\mathbf{E}} \cdot \hat{\boldsymbol{r}}^{\prime} f_{1}\left(r^{\prime}\right)\right] \quad\left(r^{\prime} \leqslant R_{V}\right) .
$$

Note that for an actual calculation of $f_{0}$ and $f_{1}$, hydrodynamic interactions can be important, since particles are now close together $r \leqslant R_{V}$, despite the fact that hydrodynamic interactions for the long-ranged correlations can be neglected.

The isotropic contribution $f_{0}\left(r^{\prime}\right)$ and the possibly non-zero diagonal elements in the tensor $\mathbf{H}$ distinguish the form in equation (28) from the more familiar form. It is well known that for dilute suspensions [5], and to within certain approximations also for higher concentrations [7-9], the function $g$ takes the form in equation (28) without the isotropic contribution $f_{0}$. However, even on the two-particle level-that is, to leading order in concentration - the equation of motion for $g$ is extremely complicated: it includes three- and four-particle correlations functions, and the equation of motion fills an entire page. In fact, in [9] the statement '... neglecting purely hydrodynamic couplings with a third particle ... ' on page 414 implies that these probably non-negligible higher-order correlation contributions are simply not considered. In [7-9] the very complicated equation of motion is considerably simplified, which may lead to an incorrect shear-rate dependence of the pair-correlation function. The higher-order correlation contributions could be responsible for the peculiar isotropic contribution in equation (28). In fact, the experiments are performed at volume fractions of about $20 \%$, where a leading-order concentration theory for the short-ranged distortions is probably not sufficient. 
Imposing the steps (i)-(iii) described above, and using equation (28) instead of step (iv), one finds, instead of equation (12),

$\begin{aligned} 0=2 D_{0} \nabla \cdot[ & \left.\left\{\beta \frac{\mathrm{d} \Pi}{\mathrm{d} \bar{\rho}}+\left|P e^{0}\right| \epsilon^{\prime}\right\} \nabla h(\boldsymbol{r})-P e^{0} \alpha^{\prime} \hat{\mathbf{E}} \cdot \nabla h(\boldsymbol{r})-\beta \Sigma \nabla \nabla^{2} h(\boldsymbol{r})\right] \\ & -\boldsymbol{\nabla} \cdot[\boldsymbol{\Gamma} \cdot \boldsymbol{r} h(\boldsymbol{r})],\end{aligned}$

where

$$
\begin{aligned}
& \epsilon^{\prime}=\frac{4 \pi}{3} \bar{\rho} \beta \int_{0}^{\infty} \mathrm{d} r r^{3} \frac{\mathrm{d} V(r)}{\mathrm{d} r} g^{e q}(r) f_{0}(r), \\
& \alpha^{\prime}=\frac{16 \pi}{15} \bar{\rho} \beta \int_{0}^{\infty} \mathrm{d} r r^{3} \frac{\mathrm{d} V(r)}{\mathrm{d} r} g^{e q}(r) f_{1}(r),
\end{aligned}
$$

represent the contributions from the isotropic and anisotropic parts of the short-ranged distortions, respectively. The contribution $\sim\left|P e^{0}\right|$ should be compared in magnitude to the leading term $\sim \beta \mathrm{d} \Pi / \mathrm{d} \bar{\rho}$ in the curly brackets in equation (29). Hence, although $P e^{0}$ is a small number, the contributions $\sim P e^{0}$ cannot be neglected, since $\mathrm{d} \Pi / \mathrm{d} \bar{\rho} \rightarrow 0$ on approach to the critical point. Fourier transformation leads to

$\lambda K_{1} \frac{\partial S(\boldsymbol{K})}{\partial K_{2}}=K^{2}\left(1+\epsilon+K^{2}\right) S(\boldsymbol{K})-K^{2}\left(1+K^{2}\right) S^{e q}(K)+\alpha K_{1} K_{2}[S(\boldsymbol{K})-1]$,

instead of equation (20), where

$$
\epsilon=\frac{|\dot{\gamma}|\left(R_{V} \xi\right)^{2}}{2 D_{0} \Sigma} \epsilon^{\prime}, \quad \alpha=\frac{|\dot{\gamma}|\left(R_{V} \xi\right)^{2}}{2 D_{0} \Sigma} \alpha^{\prime},
$$

are dimensionless groups which signify the contributions of the isotropic $(\sim \epsilon)$ and anisotropic parts $(\sim \alpha)$ of the distortion of the short-ranged part of the pair-correlation function in equation $(28)$.

Repeating the steps outlined in appendix $\mathrm{A}$ in [2] for the equation of motion (31), on readily finds

$$
\begin{aligned}
S(\boldsymbol{K})=\frac{1}{\lambda K_{1}} & \int_{K_{2}}^{ \pm \infty} \mathrm{d} X\left[\left(K^{2}-K_{2}^{2}+X^{2}\right)\left(1+K^{2}-K_{2}^{2}+X^{2}\right) S^{e q}\left(K_{1}, X, K_{3}\right)-\alpha K_{1} X\right] \\
& \times \exp \left\{-\frac{F(\boldsymbol{K} \mid X)}{\lambda K_{1}}\right\}
\end{aligned}
$$

where

$$
\begin{aligned}
F(\boldsymbol{K} \mid X)=( & \left.X-K_{2}\right)\left(K^{2}-K_{2}^{2}\right)\left(1+\epsilon+K^{2}-K_{2}^{2}\right) \\
& +\frac{1}{3}\left(X^{3}-K_{2}^{3}\right)\left(1+\epsilon+2 K^{2}-2 K_{2}^{2}\right)+\frac{1}{5}\left(X^{5}-K_{2}^{5}\right)-\frac{1}{2} \alpha K_{1}\left(X^{2}-K_{2}^{2}\right) .
\end{aligned}
$$

The upper integration limit in equation (33) is equal to $+\infty$ when $\lambda K_{1}>0$ and equal to $-\infty$ when $\lambda K_{1}<0$.

The three relevant parameters describing the response of the colloidal dispersion to an external shear flow are thus the dressed Peclet number $\lambda$ in equation (21), and the dimensionless numbers $\epsilon$ and $\alpha$ in equation (32).

Note that, contrary to the earlier prediction of (22) that $S(\boldsymbol{K})=S^{e q}(K)$ when $K_{1}=0$, we now find from equation (31) that

$$
S(\boldsymbol{K})=\frac{1+K^{2}}{1+\epsilon+K^{2}} S^{e q}(K)=\frac{\xi^{2}}{\beta \Sigma} \frac{1}{1+\epsilon+K^{2}} \quad\left(K_{1}=0\right) .
$$

This result can also be found from equations (33), (34), using the representation (A1) in [2] for the delta distribution (which can also be used to show that $S(K) \rightarrow S^{e q}(K)$ when $\dot{\gamma} \rightarrow 0$ ). 
The finite distortion in directions perpendicular to the flow direction is thus entirely due to the isotropic distortion of short-ranged correlations. The above result can be written in a more suggestive way by introducing an effective correlation length:

$$
\xi^{e f f}=\xi / \sqrt{1+\epsilon}
$$

Returning in equation (35) to the original parameters by re-substitution of the definition (19) gives

$$
S(\boldsymbol{k})=\frac{1}{\beta \Sigma} \frac{1}{\left(\xi^{e f f}\right)^{-2}+k^{2}} \quad\left(K_{1}=0\right) .
$$

This is precisely the equilibrium Ornstein-Zernike structure factor (17), except that the correlation length $\xi$ of the quiescent suspension is now replaced by a shear-rate-dependent correlation length. The effective correlation length can therefore be experimentally determined from the usual Ornstein-Zernike plot. Note, however, that this result only holds in the plane perpendicular to the flow direction. Correlation lengths in other directions are smaller than the correlation length $\xi^{e f f}$, which measures the extent of correlations in the plane perpendicular to the flow direction. The interpretation of equation (37) is as follows. The short-ranged distortions in equation (28) are responsible for the shear-induced displacement of the gasliquid critical point [4]. The effective correlation length (36) therefore reflects the larger distance to the critical point. The structure factor is still of the Ornstein-Zernike form in directions perpendicular to the flow direction as in equation (22), but now with a smaller correlation length (36) as compared to the unsheared suspension due to the larger distance to the critical point under shear flow.

\section{Experiment}

The system used in this study consists of colloidal silica spheres (102 nm diameter) grafted with stearyl alcohol, dissolved in cyclohexane. Polydimethylsiloxane (PDMS, Jansen) with a molecular weight of $206 \mathrm{~kg} \mathrm{~mol}^{-1}$ and a radius of gyration of $23 \mathrm{~nm}$ was added to induce depletion attractions between the colloidal particles, which gives rise to a gas-liquid critical point. The distance to the critical point can be tuned by gently evaporating or adding solvent. For more details on the phase behaviour of this system, see [12].

The range of the pair-interaction potential for this system is simply the sum of the diameters of the colloidal particles and the polymers, that is, $R_{V}=148 \mathrm{~nm}$.

We used a home-made cylindrical optical shear cell. The rotating inner cylinder has a radius of $21.5 \mathrm{~mm}$; the gap width is $2.47 \mathrm{~mm}$. The shear cell is placed in a cylindrical toluene bath with the second gap of the cell exactly in the middle of the bath. A $5 \mathrm{~mW} \mathrm{He-Ne} \mathrm{laser}$ (Melles-Griott) with a wavelength of $632.8 \mathrm{~nm}$ was used as a light source. The laser beam is directed along the gradient direction, so that at small scattering angles the flow-vorticity plane is probed. A pinhole is inserted in the hollow inner cylinder in order to block scattered light from the first gap. The cavity is filled with toluene to avoid refraction of the laser beam. Scattered intensities are projected on a white screen, where the laser beam passes through a hole in the middle of the screen. The size of the hole corresponds to a scattering angle of $1.4^{\circ}$ and a wavevector of $2.4 \times 10^{5} \mathrm{~m}^{-1}$. Images were taken in reflection mode with a Peltiercooled 12-bit CCD camera, with $582 \times 782$ pixels (Princeton Instruments, microMAX). The maximum scattering angle was $7.7^{\circ}$, which corresponds to a wavevector of $1.3 \times 10^{6} \mathrm{~m}^{-1}$.

Scattering patterns were recorded for six different correlation lengths $(313,650,682,747$, 1000 , and $1460 \mathrm{~nm})$. The shear rate was varied between $\dot{\gamma}=0$ and $37 \mathrm{~s}^{-1}$. This corresponds for our system to a maximum value of the bare Péclet number of about 0.2. 


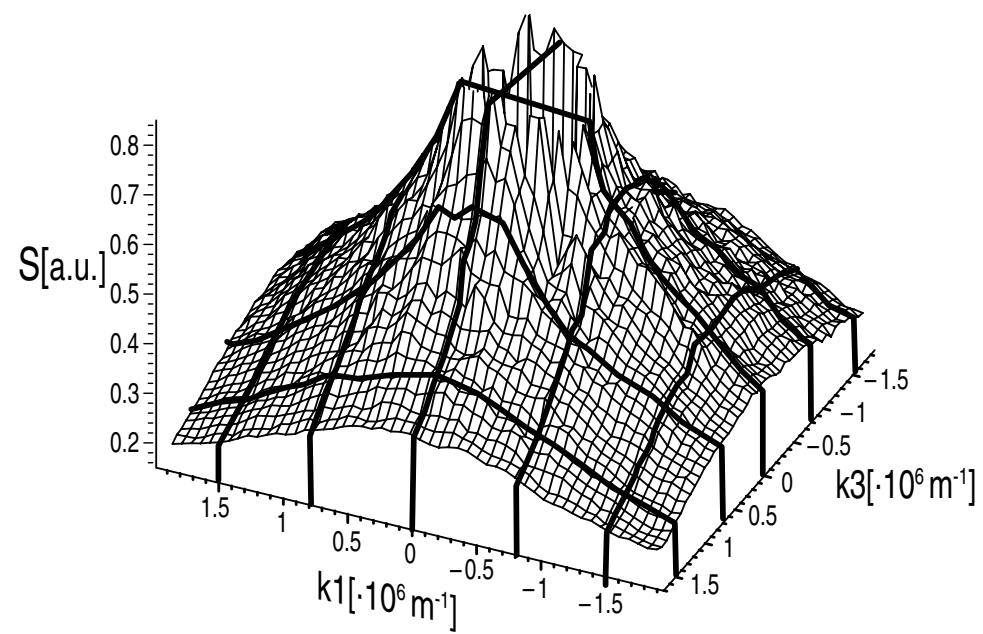

Figure 2. The equilibrium structure factor for the correlation length $650 \mathrm{~nm}$. The thick curves indicate typical cross-sections that are used for data analysis.

\subsection{Data analysis and results}

For convenience of data analysis, we extract data for ten cross-sections from each scattering pattern: five cross-sections at fixed values of $K_{1}$ as functions of $K_{3}$, and five cross-sections at fixed $K_{3}$ as functions of $K_{1}$. These cross-sections are indicated by the thick lines in figure 2, where an experimental, equilibrium Ornstein-Zernike structure factor is plotted. In accordance with theory, the scattered intensities for cross-sections are invariant under coordinate inversion to within experimental error, so these can be averaged. This then results in six scattering curves per scattering pattern, which are compared to the theory described above.

Since no absolute intensity measurements have been performed, experimental data for the shear-induced changes of the scattered intensities are compared to the theoretically predicted structure factors (equations (33), (34)):

$$
\Delta S^{\star}(\boldsymbol{K}) \equiv \frac{\beta \Sigma}{\xi^{2}}\left\{S^{e q}(K)-S(\boldsymbol{K})\right\}=\frac{I^{e q}(K)-B}{A^{e q}}-\frac{I(\boldsymbol{K})-B}{A},
$$

where $I^{e q}$ and $I$ are the scattered intensities in arbitrary units without and with shear flow, respectively. Furthermore, $B$ is a wavevector-and shear-rate-independent background intensity that arises from stray light and reading noise of the CCD camera. This quantity is measured from a scattering experiment where the cell is filled with just solvent. The 'scattering contrasts' $A^{e q}$ and $A$ are factors of proportionality between experimental intensities and the structure factor without shear flow and with shear flow, respectively. Unlike the background $B$, the scattering contrast $A$ is shear-rate dependent as a result of the shear-rate dependence of the turbidity: on shearing a near-critical suspension, its turbidity decreases [13], so the incident and the scattered intensity are less attenuated, leading to an increase of $A$ with increasing shear rate. The prefactor $\beta \Sigma / \xi^{2}$ is factored out in the definition (38) of $\Delta S^{\star}$, because the expression in equation (33) for the structure factor is proportional to $\xi^{2} / \beta \Sigma$, and no absolute intensity measurements have been made. Note that at these small wavevectors, the form factor of the colloidal particles is virtually equal to unity.

The quantities $\xi$ and $A^{e q}$ are determined from a least-squares fit of the wavevector dependence of the Ornstein-Zernike structure factor in equation (17) to the form $\left(I^{e q}-B\right) / A^{e q}$. The corresponding Ornstein-Zernike plots for equilibrium suspensions are given in figure 3 . 


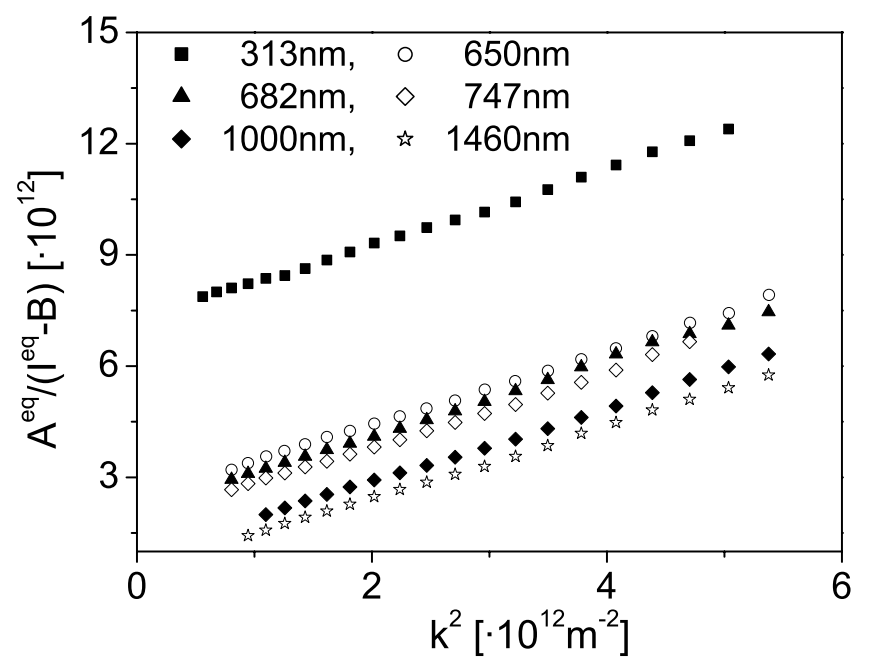

Figure 3. Ornstein-Zernike plots from which correlation lengths are determined. The correlation lengths corresponding to the different curves are indicated in the upper left part of the figure.

For a given equilibrium correlation length, the wavevector dependence of the shear-induced distortion $\Delta S^{\star}$ for all applied shear rates is then fitted with respect to the parameters $A, \lambda / \dot{\gamma}$, and $\epsilon / \dot{\gamma}$. Including the parameter $\alpha$ did not significantly improve the comparison with theory, so this parameter is set equal to 0 in the following. It should be emphasized that for a given equilibrium correlation length, the entire wavevector dependence for a whole set of shear rates is fitted with just two shear-rate-independent theoretical parameters $(\lambda / \dot{\gamma}$ and $\epsilon / \dot{\gamma})$ and a single, shear-rate-dependent experimental parameter $(A)$.

Figures 4 and 5 show typical least-squares comparisons between theory and experiment for two shear rates for the correlation lengths $\xi=650$ and $1460 \mathrm{~nm}$, respectively. The dotted curve is the best comparison with theory where short-ranged distortions are neglected - that is, $\epsilon$ is set equal to zero-while the solid curve shows the full structure factor as given in equations (33), (34) (with $\alpha=0$ ). The finite distortion of the structure factor in directions perpendicular to the flow direction is most clearly seen in the top-left-corner plots in these figures, which are cross-sections where $k_{1}=0$. As explained in the previous section (see equation (22)), without contributions from shear-induced distortions of short-ranged correlations $(\epsilon=0)$, theory predicts no effect of shear flow. Clearly, the effects of distortions of short-ranged correlations are essential to explain our experimental data on shear-induced long-ranged correlations. Moreover, the theory describes the experiments quantitatively correctly. In particular, the Ornstein-Zernike-like wavevector dependence in directions perpendicular to the flow direction as predicted by equations (36), (37) is confirmed. The fact that the data points are somewhat above the theoretical predictions in the middle figures is due to the contribution of additional stray intensities at small angles, close to the laser beam. Note that the predicted shallow minimum in the scattering curves at fixed $k_{3}$ as functions of $k_{1}$ at $k_{1}=0$ is also seen experimentally, provided that $k_{3}$ is large enough for us to be able to fully neglect stray light intensity from the incident beam.

Equations (21), (32) predict that $\lambda / \dot{\gamma}$ and $\epsilon / \dot{\gamma}$ are $\sim \xi^{4}$ and $\sim \xi^{2}$, respectively. This is indeed what is found, as shown in figure 6 . The slopes of the straight lines in figure 6 are equal to $4.4 \pm 0.5$ and $2.3 \pm 0.4$, respectively, which confirms the theoretical predictions. From the linear fits in figure 6, values for the prefactors $\beta \Sigma / R_{V}^{2}$ and $\beta \epsilon^{\prime}$ in equations (21), (30) 

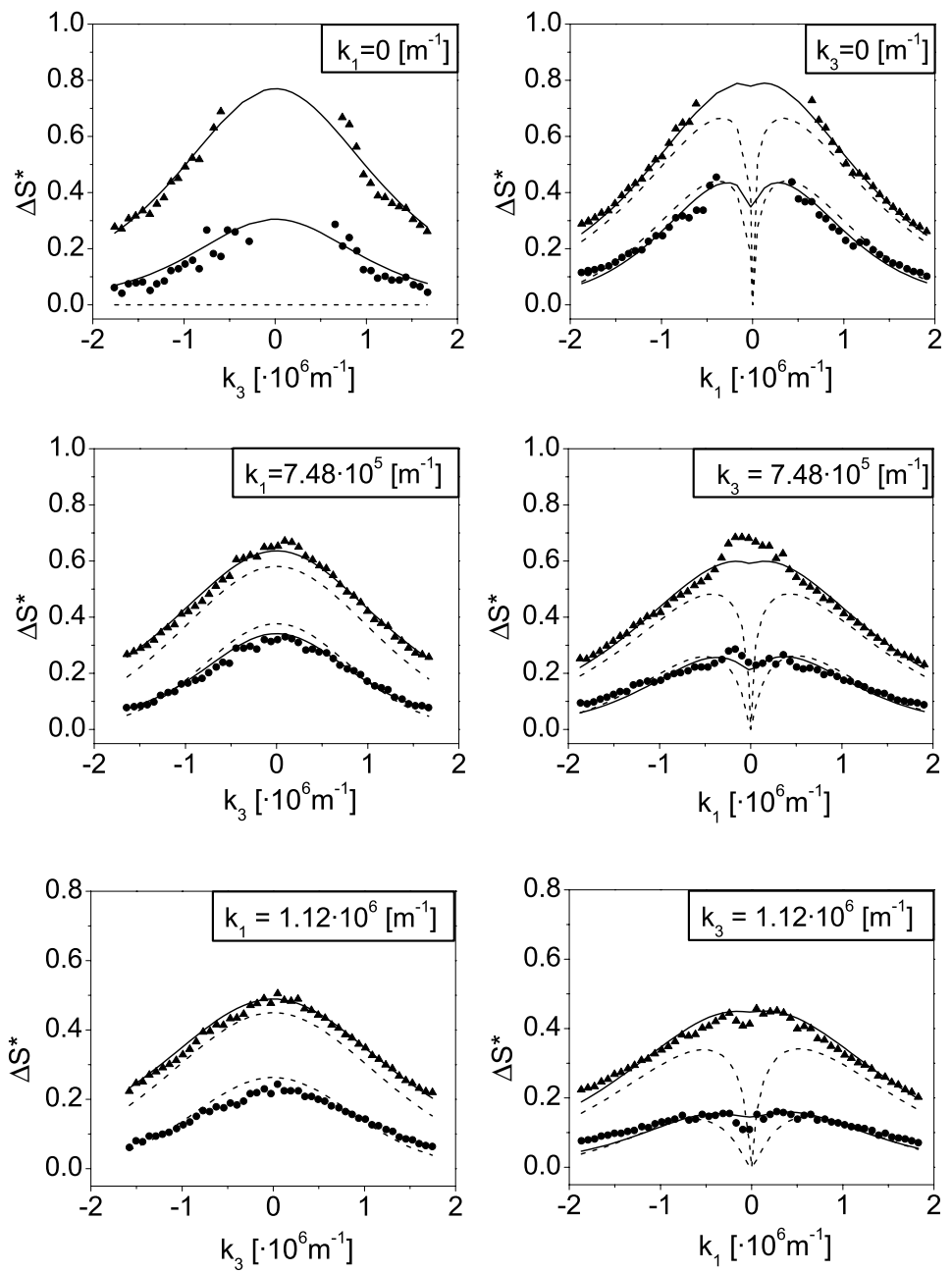

Figure 4. Typical fitting results for a correlation length $650 \mathrm{~nm}$ for the two shear rates $\dot{\gamma}=33.5 \mathrm{~s}^{-1}$ (upper curves) and $4.4 \mathrm{~s}^{-1}$ (lower curves). The values for the wavevectors indicated in the figures are the wavevectors where the cross-sections are taken, as indicated by the thick curves in figure 2 . The points are experimental data, the dotted curve is the best fit to the older theory, where distortion of short-ranged correlations is neglected, while the solid curves are best-fit results obtained with the new theoretical results of equations (33), (34). Note the difference in scale of the vertical axis between the different cross-sections.

can be extracted. It is found that $\beta \Sigma / R_{V}^{2}=0.78 \pm 0.07$ and $\epsilon^{\prime}=1.00 \pm 0.08$. This value for $\beta \Sigma / R_{V}^{2}$ is of the same order as a crude, theoretical estimate $[1,2,14]$.

The expected increase of the scattering contrast $A$ with increasing shear rate is also observed, as shown in figure 7 for a few correlation lengths. Moreover, as the correlation length increases - that is, on closer approach to the critical point - the scattering contrast becomes a stronger function of the shear rate, and ultimately develops a non-analytic dependence at small shear rates, in accordance with what is known for the turbidity [13].

We also performed least-squares fits with respect to the shear-rate-independent parameter $\epsilon / \dot{\gamma}^{\nu}$, for various values of the exponent $\nu$. Such fits correspond to expansions of the form of equation (28) where $P e^{0}$ is replaced by $\left(P e^{0}\right)^{\nu}$. In figure 8, the minimum standard deviation 

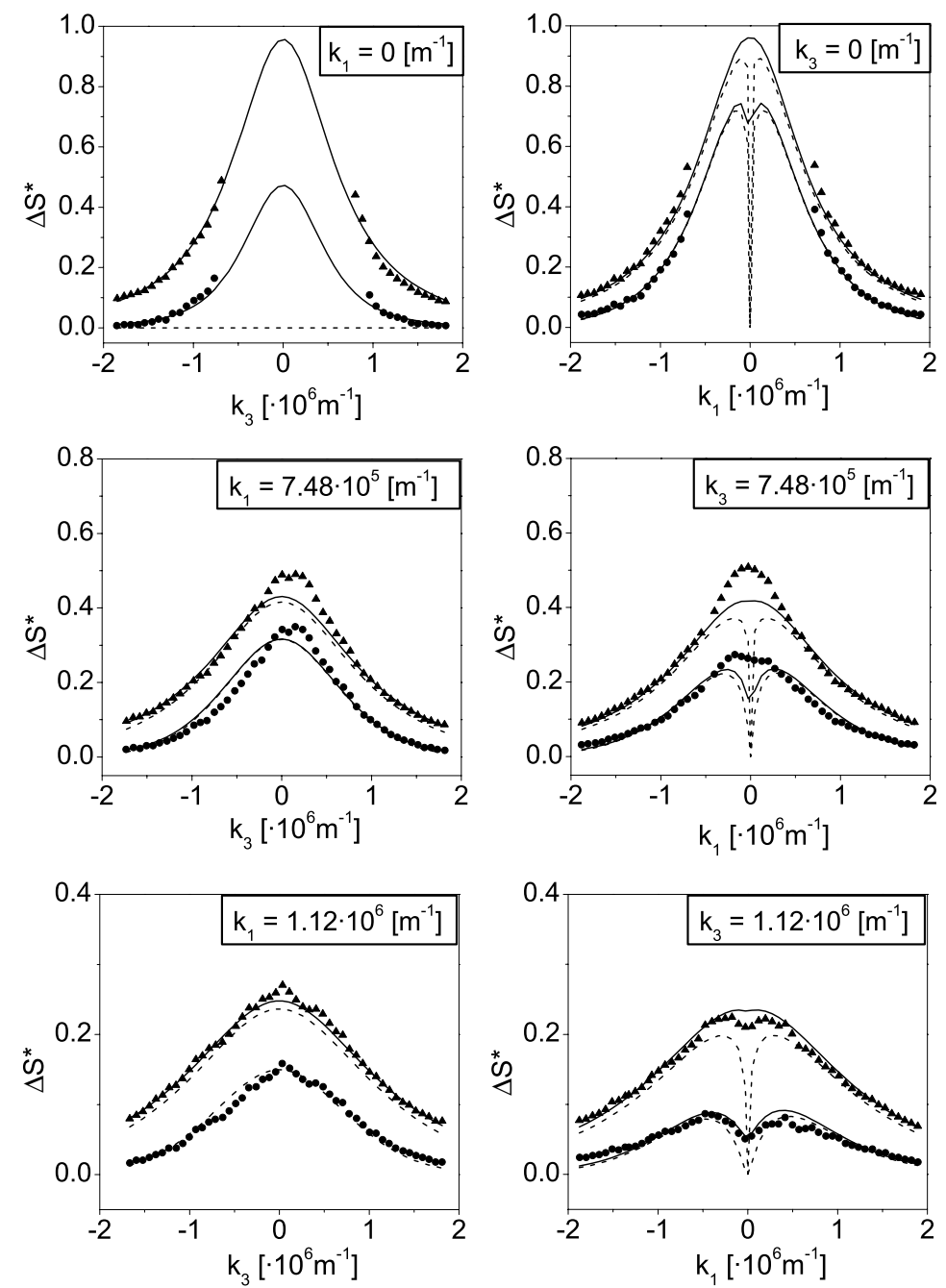

Figure 5. As figure 4, but now for a correlation length of $1460 \mathrm{~nm}$. The shear rates are $\dot{\gamma}=27.5 \mathrm{~s}^{-1}$ (upper curves) and $1.1 \mathrm{~s}^{-1}$ (lower curves). Note the difference in scale of the vertical axis between the different cross-sections.

for these fits is plotted as a function of the value of the exponent $v$. Clearly, best fits are obtained for $v=1( \pm 0.2)$. Since the equation of motion for the pair-correlation function, even at low concentrations, is extremely complicated, it is a formidable task to actually deduce an expansion of the pair-correlation function of the form of equation (28) from this equation of motion, especially at somewhat higher concentrations (our samples have a volume fraction of colloidal spheres of about 20\%). The experimental evidence for the expansion equation (28) for the short-ranged part of the pair-correlation function is indirect, since it is verified through its effect on the distortion of long-ranged correlations. Scattering data at large wavevectors on the same system would be desirable to verify equation (28) more directly. 


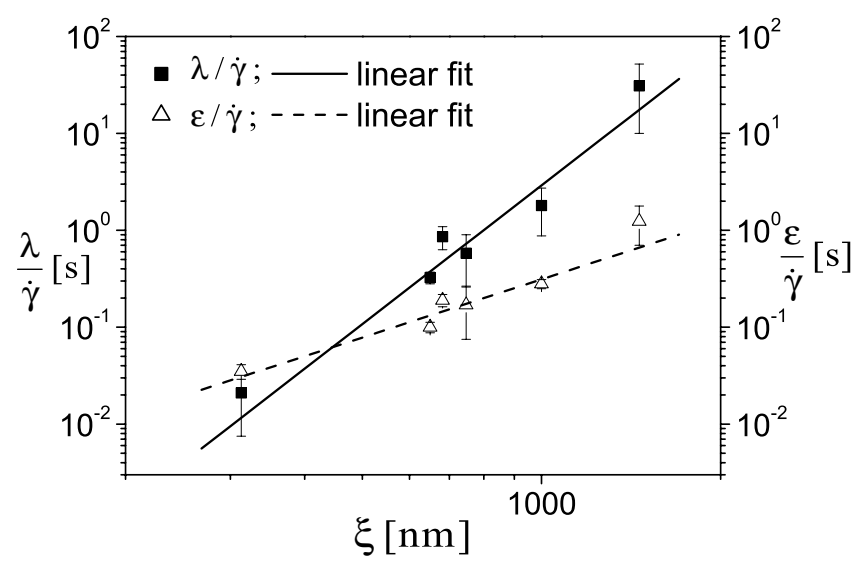

Figure 6. The parameters $\lambda / \dot{\gamma}$ and $\epsilon / \dot{\gamma}$ obtained from the fitting procedure as a function of the correlation length.

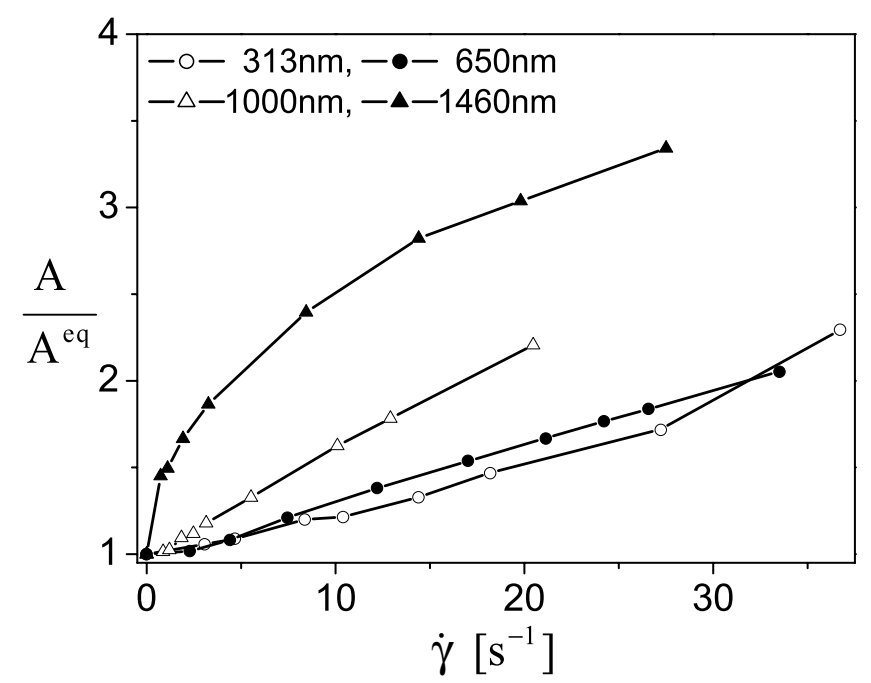

Figure 7. The shear-rate dependence of the scattering contrasts for four correlation lengths as indicated in the figure.

\section{Summary and conclusions}

The aim of this study was to investigate the response of a near-critical colloidal dispersion to a stationary shear flow. Starting from the $N$-particle Smoluchowski equation, an equation of motion for the long-range behaviour of the pair-correlation function in the vicinity of the gas-liquid critical point under shear flow conditions was derived. The importance of the short-ranged distortion of the pair-correlation function was recognized, as a result of the experimentally observed significant distortion of the structure factor at small wavevectors in the flow-vorticity plane in directions perpendicular to the flow direction. The assumed form (28) for the shear-rate dependence of the short-ranged behaviour of the pair-correlation function, which is needed to explain our small-angle light scattering data, could be tested more directly by means of scattering experiments at larger wavevectors. 


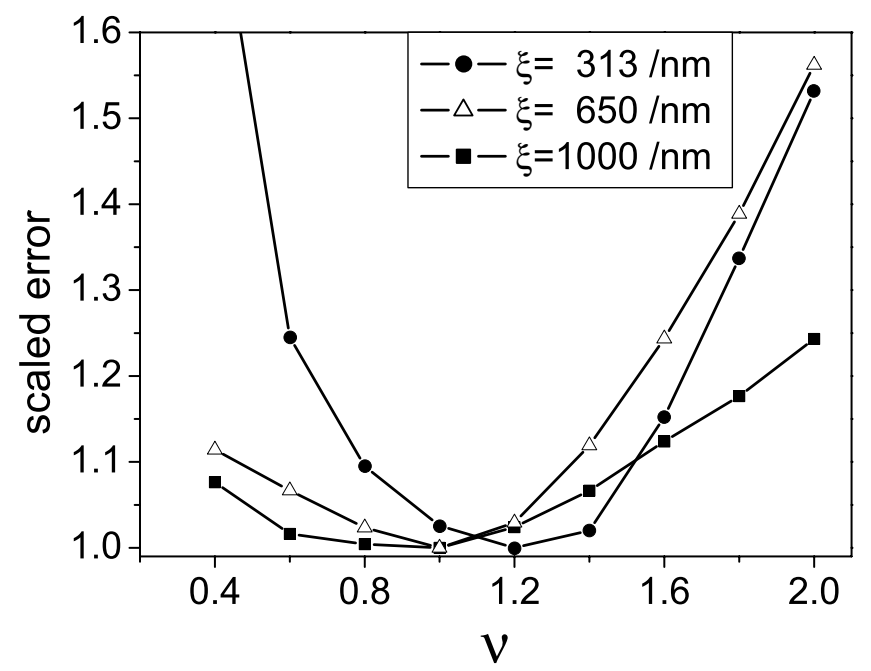

Figure 8. The minimum standard deviation from least-squares fits for various values of the exponent $v$, for three different correlation lengths as indicated in the figure. The error here is defined as $\frac{1}{n} \sum_{j}\left(Y_{j}^{\exp }-Y_{j}^{t h}\right)^{2}$, where $n$ is the number of data points involved in the fit, $Y_{j}^{\exp }$ is the $j$ th experimental value for the structure factor, and $Y_{j}^{t h}$ is the corresponding fitted, theoretical value. Errors are scaled with respect to the minimum standard deviation as a function of $v 1$.

Earlier quantitative studies on the effect of shear flow on critical correlations are concerned with integrated properties such as the critical divergence of the shear viscosity [15], the shearrate dependence of the turbidity [13], and the critical behaviour of flow-induced dichroism [12]. These integrated quantities were shown to agree with the theory where distortions of shortranged correlations are neglected (except for the shear-induced dichroism on close approach to the critical point). These integrated quantities are not very sensitive to the effect that shortranged distortions have on long-ranged distortions. This can be seen from the plots on the righthand side in figures 4,5 . The volume in wavevector space where there is a difference between the older and the extended theory (the dotted and solid curves, respectively) is relatively small, the more so on closer approach to the critical point. Differences between the two theories are only significant within a narrow region in wavevector space extending in the flow direction. In addition, the integrands of the wavevector integrals that represent these integrated properties are small for small wavevectors. This explains the quantitative agreement found before for these integrated quantities with the theory where short-ranged distortions are neglected.

Taking short-ranged distortions into account gives rise to two new parameters ( $\epsilon$ and $\alpha)$ in the stationary equations of motion (33), (34) for the structure factor. Experimental data are quantitatively described by an extended theory where these short-ranged correlations are accounted for to leading order in the bare Peclet number (10). A key feature of the new expressions of equations (33), (34) for the structure factor is that in the plane perpendicular to the flow direction (where $K_{1}=0$ ), the structure factor significantly decreases with increasing shear rate, the more so on closer approach to the critical point. In contrast, the previous theory, where shear-induced distortions of short-ranged correlations were neglected, predicts no shear-induced distortion in this plane; that is, in directions perpendicular to the flow direction the structure factor attains its equilibrium Ornstein-Zernike form. In fact, as shown in [4], distortions of short-ranged correlations are responsible for the shear-induced shift of the gasliquid critical point. One might interpret the effective correlation length in equation (36) as 
the actual correlation length that is diminished due to the shear-induced displacement of the location of the critical point.

The decrease of intensity in directions perpendicular to the flow direction implies that the critical point is shifted into the region where the system would be unstable in the absence of shear flow. This has also been observed for the upper critical point in various low-molecularweight polymer systems [17-20], where the critical point shifts to lower temperatures on applying shear flow, and in binary fluid mixtures [21-23]. An opposite displacement of the critical point has been observed in high-molecular-weight polymer systems [20]. Here the shear-induced deformation of the polymer coil itself may play an important role. Such singleparticle deformations are of course not included in the present theory, and are certainly absent in our experimental colloidal system of rigid spheres mixed with relatively small polymers. The absence of a shear-induced shift of phase transition lines in a polymer system at low shear rates [24] is an indication that shift is measured by the bare Peclet number also for polymers. An overview on flow-induced mixing, demixing, and phase transitions in polymeric fluids is given in [25].

The origin of the linear (but non-analytic) isotropic distortion of short-ranged correlations in equation (28) remains an open issue. The possibly incorrect prediction of the shearrate dependence of the pair-correlation function on using simplified equations of motion, as mentioned in section 2, might be the reason for the discrepancy between the analytical results as derived by Brady and Vicic in [26] for the shear-rate dependence of bulk stresses and the computer simulation results of Rastogi et al in [27]. If three- and four-particle correlation functions in the equation of motion for the pair correlation to leading order in density are indeed responsible for the peculiar shear-rate dependence, it seems quite impossible to ever resolve this issue analytically. Computer simulations and experiments are needed to verify equations such as (28). Since $f_{0}$ is found to be independent of the distance from the critical point, it seems that the form (28) is not specific to systems close to their gas-liquid critical point. We are planning to perform neutron scattering experiments under shear flow on the same polymer-colloids mixtures as were used in the present study, in order to examine more directly the shear-rate dependence of the molecular, short-ranged part of the pair-correlation function, with an emphasis on the isotropic contribution to the distortion.

\section{References}

[1] Dhont J K G 1996 An Introduction to Dynamics of Colloids (Amsterdam: Elsevier)

[2] Dhont J K G and Nägele G 1998 Phys. Rev. E 587710

[3] Dhont J K G and Bodnár I 1998 Phys. Rev. E 584783

[4] Dhont J K G 1996 Phys. Rev. Lett. 224269

[5] Batchelor G K 1977 J. Fluid Mech. 8397

[6] Ronis D 1984 Phys. Rev. A 291453

[7] Brady J F 1993 J. Chem. Phys. 99567

[8] Wagner N J and Russel W B 1988 Physica A 155475

[9] Lionberger R A and Russel W B 1997 J. Chem. Phys. 106402

[10] Szamel G 2001 J. Chem. Phys. 1148708

[11] Fixman M 1960 J. Chem. Phys. 331357

[12] Lenstra T A J and Dhont J K G 2001 Phys. Rev. E 6361401

[13] Verduin H and Dhont J K G 1995 Phys. Rev. E 521811

[14] Fixman M 1964 Adv. Chem. Phys. 6715

[15] Bodnár I and Dhont J K G 1996 Phys. Rev. Lett. 775304

[16] Lenstra T A J, Dogic Z and Dhont J K G 2001 J. Chem. Phys. 11410151

[17] Silberberg A 1952 Thesis University of Johannesburg

Silberberg A and Kuhn W 1952 Nature 170450

Silberberg A and Kuhn W 1954 J. Polym. Sci. 1321 
[18] Hashimoto T, Takebe T and Asakawa K 1993 Physica A 194A 338

[19] Fujioka K, Takebe T and Hashimoto T 1993 J. Chem. Phys. 98717

[20] Wolf B A 1984 Macromolecules 17615

[21] Beysens D, Gbadamassi M and Boyer L 1979 Phys. Rev. Lett. 431253

[22] Beysens D, Gbadamassi M and Moncef-Bouanz B 1983 Phys. Rev. A 282491

[23] Fukuhara K, Hamano K, Kuwahara N, Sengers J V and Krall A H 1993 Phys. Lett. A 176344

[24] Wu X-L, Pine D J and Dixon P K 1991 Phys. Rev. Lett. 182408

[25] Larson R G 1992 Rheol. Acta 31497

[26] Brady J F and Vicic M 1995 J. Rheol. 39545

[27] Rastogi S R, Wagner N J and Lustig S R 1996 J. Chem. Phys. 1049234 